\title{
Treatment of Pulp and Paper Mill Wastewater with Various Molecular Weight of PolyDADMAC Induced Flocculation with Polyacrylamide in the Hybrid System
}

\author{
M. A. A. Razali ${ }^{1,2}$, Z. Ahmad ${ }^{1}$, A. Ariffin ${ }^{1 *}$ \\ ${ }^{1}$ School of Material and Mineral Resources Engineering, Engineering Campus, Universiti Sains Malaysia, Nibong Tebal, Penang, \\ Malaysia \\ ${ }^{2}$ Research and Development Department, Global Network Technology SdnBhd, Bentong, Malaysia \\ Email: *azlan@eng.usm.my
}

Received August 1, 2012; revised September 4, 2012; accepted September 12, 2012

\begin{abstract}
Flocculation studies between dual polymers on pulp and paper mill wastewater are reported in this paper. The effects of different molecular weights of polyDADMAC and different dosages of Polyacrylamide (PAM) were studied. The molecular weights of polyDADMAC used were $8.8 \times 10^{4}, 10.5 \times 10^{4}$ and $15.7 \times 10^{4} \mathrm{~g} / \mathrm{mol}$. The flocculation performance was analyzed in jar tests with PolyDADMAC and Polyacrylamide dosages ranging from $0.4-2.0 \mathrm{mg} \cdot \mathrm{L}^{-1}$ and $0.4-8.0$ $\mathrm{mg} \cdot \mathrm{L}^{-1}$ respectively. A higher molecular weight and a $6.0 \mathrm{mg} \cdot \mathrm{L}^{-1}$ dosagepolyDADMAC gave the highest level of flocculation based on turbidity and TSS removal. In addition, increasing the molecular weight of PolyDADMAC increased $\zeta$ potential values approaching zero. This indicated that polyDADMAC acts as a destabilizer. Based on TSS, the addition of PAM will improve the size of microflocs created by polyDADMAC. It demonstrates that PAM acts as a bridger between microflocs.
\end{abstract}

Keywords: Flocculation; PolyDADMAC; Polyacrylamide; Zeta Potential; Molecular Weight

\section{Introduction}

Much research has concluded that flocculation is an efficient and cost effective method for water and wastewater treatment [1-4]. Flocculation can be defined as a process that makes finely dispersed particles aggregate and form large floc [5]. Flocculation is promoted by the addition of organic or inorganic chemicals. In recent years, the use of synthetic polyelectrolytes as flocculants for the removal of suspended solids in wastewater treatment has become a widely used practice.

There have been several studies on hybrid polyelectrolytes systems to improve the flocculation of suspended particles in wastewater [6-9]. Dual flocculants often offer advantages over a single component flocculant, such as better control of flocculation kinetics and improved floc strength. According to $\mathrm{Yu}$ and Somasundaran [6], flocculants showed limited flocculation while being used alone, but achieved good flocculation when used together. Chitikela and Dentel [10] propose that hybrid cationic polyelectrolyte conditioning is more effective than single polyelectrolytes. These dual flocculant approaches were attempts by different branches of industry, both mineral

${ }^{*}$ Corresponding author. processing [11-13] and paper making [14,15].

The first research on this topic was conducted by Britt [16] in regards to the paper making industry. He observed that a dual flocculant promoted higher flocculation, but the aggregates were very fragile and, hence, easily redispersed by an applied shear. To overcome this problem, a new technique was tested by using a combination of cationic and anionic polymers. The cationic polymer was used to flocculate the slurry to the point where no solids were detected in the supernatant, followed by the addition of an anionic polymer. Results from this technique exhibited increased firmness when compared to the single polymer test. Most previous studies explored a combination of cationic and anionic polymers, with the exclusion of Swerin et al. [17], who studied the absorbtion and flocculation rates when using two cationic polymers. They used different molecular weights of polyDADMAC and PAM, but only covered the flocculation process on microcrystalline cellulose (MCC). To date, there are no studies of different of molecular weight of polyDADMAC and PAM for pulp and paper mill wastewater particles.

The pulp and paper mill industry consumes a large amount of water and therefore discharges a large amount 
of wastewater [18]. The large amount of wastewater constitutes one of the major sources of aquatic pollution. The bleaching process produces the largest volume of pollutants, which generates several chlorinated compounds via chlorination and others toxic organic compounds such as lignin. Highly toxic materials are formed from lignin and its derivatives, while recalcitrant compounds are responsible for the high BOD and COD [19]. In addition to being highly toxic, lignin and its derivatives are also mutagenic [20]. Lignin causes death to zooplankton and fish, and profoundly affects the terrestrial ecosystem [21]. Therefore, it is obligatory that the effluent from pulp and paper mills is treated before entering receiving waters.

This study employs different molecular weights of cationic polyDADMAC and different dosages of emulsion polyacrylamide in pulp and paper mill wastewater samples. The objective of this work was to investigate the synergistic effects of addition of different molecular weights of polyDADMAC on the flocculation of pollutants present in pulp and paper mill wastewater using a constant molecular weight of emulsion polyacrylamide. $\zeta$ potential of the supernatant, the turbidity, total suspended solids (TSSs) and chemical oxygen demand (COD) concentrations were measured as the evaluating parameters.

\section{Experimental}

\subsection{Materials}

Pulp and paper mill wastewater was collected from the wastewater treatment plant equalization tank of a paper mill in Penang, Malaysia, in accordance with ASTM E 300-03. This factory produces about 3000 metric tons of tissue paper a month and produces $96 \mathrm{~m}^{3}$ of wastewater per ton of paper produced. The characteristics of the wastewater collected from the factory are similar to those found in previous studies [20,21]. The polyDADMAC flocculants used in this study were radically synthesized with various monomer concentrations. The molecular weights were obtained by synthesizing various monomer concentrations, as shown in Table 1. The molecular weights were calculated based on intrinsic viscosity values. The $\zeta$ potential of each polyDADMAC at $\mathrm{pH} 7$ were determined and also shown in Table 1. The cationic polyacrylamides (PAM) used in this study were supplied by Global Network Technology SdnBhd, Malaysia. Molar mass of this PAM is $10 \mathrm{MDa}$. Distilled water was

Table 1. Molecular weight and $\zeta$ potential for polyDADMAC samples.

\begin{tabular}{ccc}
\hline Prepared samples & Molecular weight $(\mathrm{g} / \mathrm{mol})$ & $\zeta$ potential $(\mathrm{mV})$ \\
\hline PDM01 & $8.8 \times 10^{4}$ & 40.15 \\
PDM02 & $10.5 \times 10^{4}$ & 42.10 \\
PDM03 & $15.7 \times 10^{4}$ & 48.60 \\
\hline
\end{tabular}

used to prepare all the polyDADMAC and emulsion Polyacrylamide feedstock solutions of $0.1 \%$ respectively.

\subsection{Experimental Procedure}

A jar test was performed with the conventional jar apparatus (VelpScientifica FC6S model) using 500-ml wastewater samples. Different combinations of PolyDADMAC molecular weights (as shown in Table 1), polyDADMAC dosages $\left(0.4,0.8,1.2,1.6,2.0 \mathrm{mg} \cdot \mathrm{L}^{-1}\right)$ and PAM dosages $\left(0.4,2,4,6\right.$ and $\left.8 \mathrm{mg} \cdot \mathrm{L}^{-1}\right)$ were tested. The selected PolyDADMAC dosage and Molecular weight was added to $500 \mathrm{ml}$ of wastewater and stirred for a period of $2 \mathrm{~min}$ at $200 \mathrm{rpm}$. This was followed by slow mixing for $10 \mathrm{~min}$ at $35 \mathrm{rpm}$. Selected polyacrylamide dosages were then introduced into the wastewater samples 45 seconds after the polyDADMAC additions. The flocs formed were allowed to settle for $5 \mathrm{~min}$. After settling, the turbidity, TSSs, Zeta potential and COD of the supernatants were determined. The experiments were repeated several times to obtain an average value.

\subsection{Analytical Techniques}

COD was evaluated using COD vials (Hach, United States) with different sensitivity ranges. Sample digestion was performed in a DRB200 reactor (Hach) over 2 hours at $150^{\circ} \mathrm{C}$. Solutions being tested for sample digestion were cooled at room temperature before being measured by a DRB200 Digital Reactor, 15-Wells (Hach). The supernatant turbidity before filtration was measured with a turbidity meter (from Lovibond). A pH meter (CyberScan model, Eutech Instruments, Singapore) was used to measure the $\mathrm{pH}$ of the solutions. The TSS concentration was determined by filtering a well-mixed sample through a glass fiber filter (GA 55, Advantec, Japan), and the residues retained on the filter were dried in an oven at $103^{\circ} \mathrm{C}$ for $60 \mathrm{~min}$ prior to weighing. The $\zeta$ potential was determined with a Malvern Mastersizer 2000.

\section{Results and Discussions}

In this study, it was hypothesized that polyDADMAC would perform as a charge neutralizer while PAM would perform as a bridger. The addition of polyDADMAC into the pulp and paper mill wastewater was proven to destabilize the stability of negatively charged particles. The destabilization process caused particle-particle attraction and created microflocs. The incorporation of PAM to the system immediately increased the size of flocs. Loop and tails in PAM chains were able to absorb into particles and create bridges between particles, thus increasing the size of microflocs, as shown in Figure 1(c). Figure 1 is a video caption of the above mentioned flocculation process. 


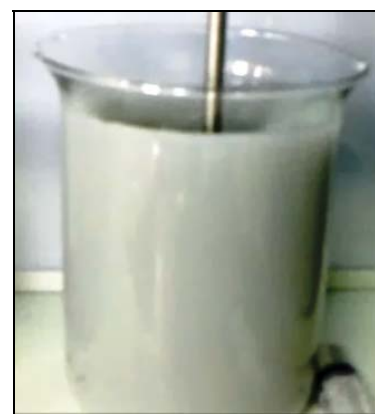

(a)

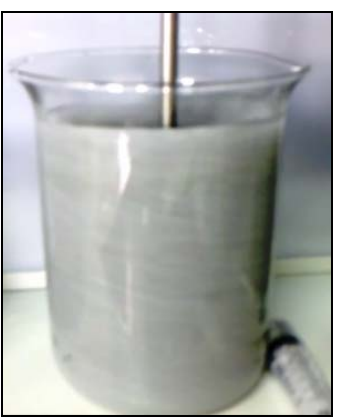

(b)

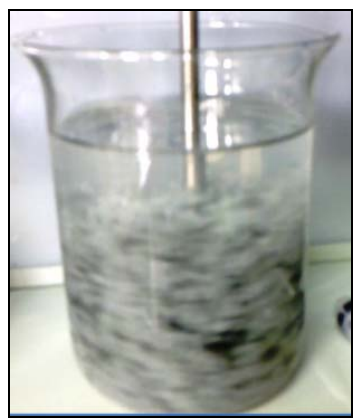

(c)

Figure 1. Pulp and paper mills wastewater: (a) Without any polymers; (b) Addition of polyDADMAC; (c) Addition of polyacrylamide.

\subsection{Effect on Turbidity Removal}

The influence of the molecular weight of PolyDADMAC, with several PAM dosages, on turbidity removal is shown in Figure 2. Generally, turbidity removal increased with an increasing molecular weight. At the 0.4 $\mathrm{mg} \cdot \mathrm{L}^{-1}$ dosage of PAM, PDM03 showed higher flocculation turbidity removal than PDM01 and PDM02, which were lower in molecular weight. This observation was thought to be caused by the contribution of polyDADMAC chains. Chains of polymer are directly related to the repeating unit, which at high molecular weights of polyDADMAC gave longer chains and many repeating units of DADMAC. In addition, each repeating unit of DADMAC brings one positive charge. According to Subramanian (1999), it has been accepted that one of the factors of the flocculation process is charge neutralization [22]. Increasing repeating units will increase the positive charge, thus improving the charge neutralization or destabilization of negative particles. Increasing the molecular weight also allows for larger loops and ends to develop, and therefore yeilds more space to attract suspended particles [23]. This phenomenon was consistentwith the study by Zang et al. [24] on reed pulp suspension. Zang observed that increasing the positive charge increased flocculation efficiency.

Figure 2 also shows that high level flocculation andoptimal dosing for this system occurred at $1.2 \mathrm{mg} \cdot \mathrm{L}^{-1}$ of polyDADMAC dosage. A dosage lower than $1.2 \mathrm{mg} \cdot \mathrm{L}^{-1}$ can cause a decrease in turbidity removal (gap between lowest and highest molecular weight) with an increase in PAM dosage, as shown in Table 2. This phenomenon may be due to charge repulsion between both polyelectrolytes. Initially, at a low dosage of PAM, the negative charge of particles is destabilized by polyDADMAC. The addition of PAM creates bridges between microflocs produced by polyDADMAC. Increasing the polyDADMAC molecular weight improved the efficiency of flocculation due to an increase in negative charge destabilization. After a certain dosage of PAM, saturation occured. Excessive amounts of PAM create charge repulsion, thus reducing the level of flocculation. This phenomenon is illustrated in Figure 3. According to Lee [25], excessive additional PAM doses seem to deteriorate inter-particle flocculation efficiencies which contribute from conformational changes of absorbed PAM molecules on particles surfaces.

\subsection{Effect on Total suspended solids}

The removal of TSSs from pulp and paper mill wastewater, following treatment with different molecular weights

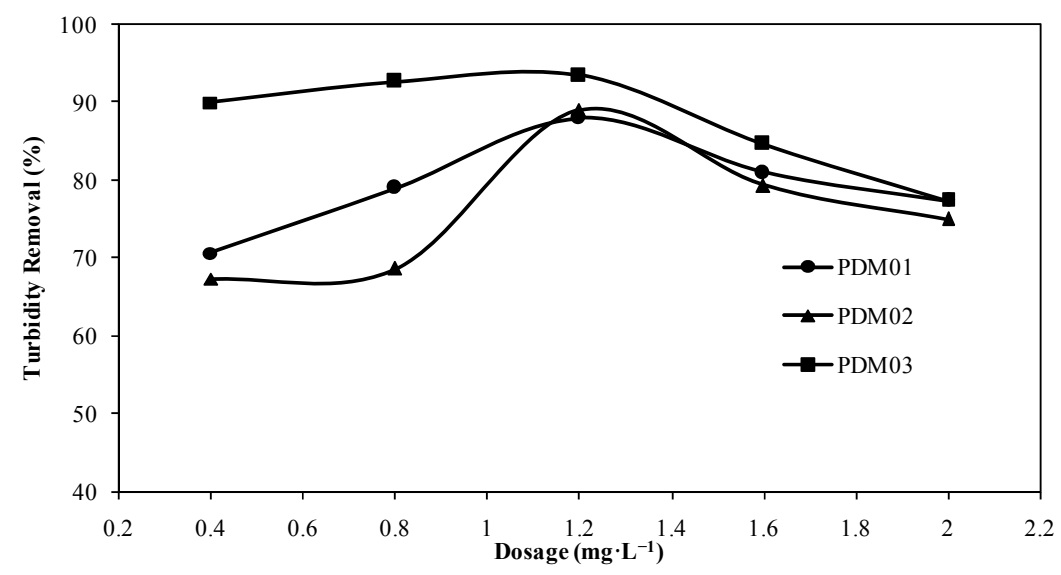

(a) 


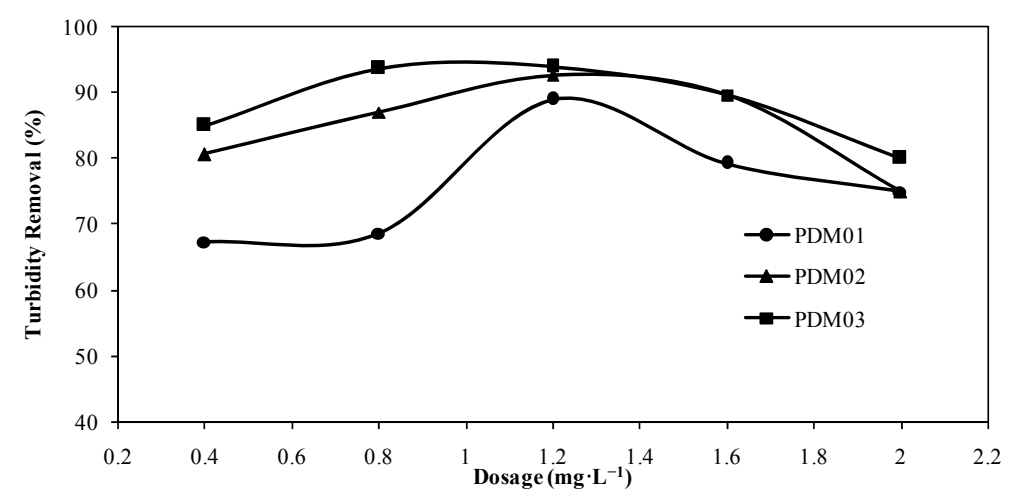

(b)

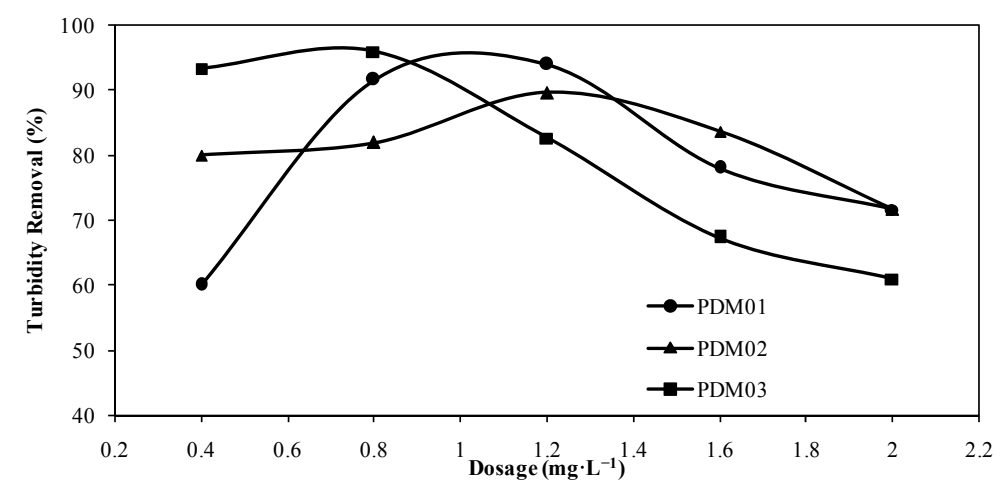

(c)

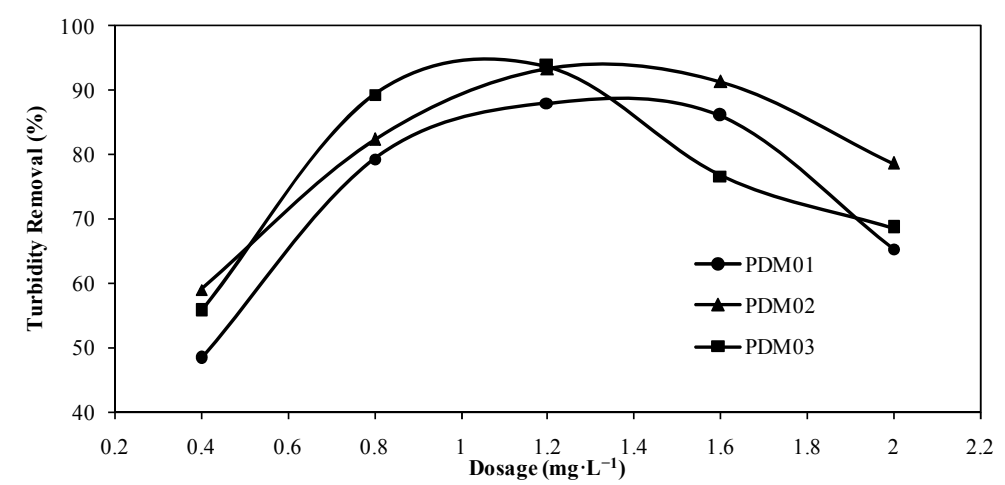

(d)

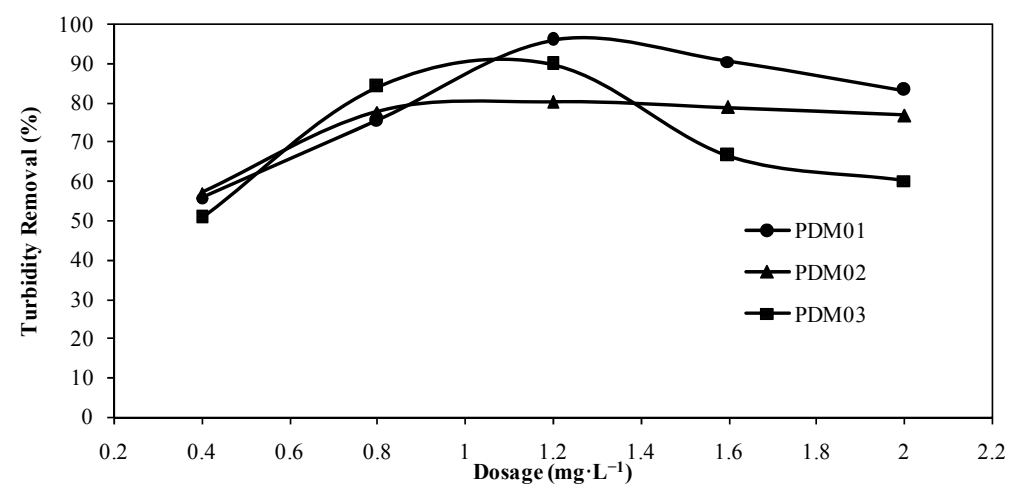

(e)

Figure 2. Effect of different molecular weight of PolyDADMAC on turbidity removal at (a) 0.4; (b) 2.0; (c) 4.0; (d) 6.0; and (e) 8.0 PAM dosages $\left(\mathrm{mg} \cdot \mathrm{L}^{-1}\right)$. 
Table 2. Turbidity removal deviation at different PAM dosage.

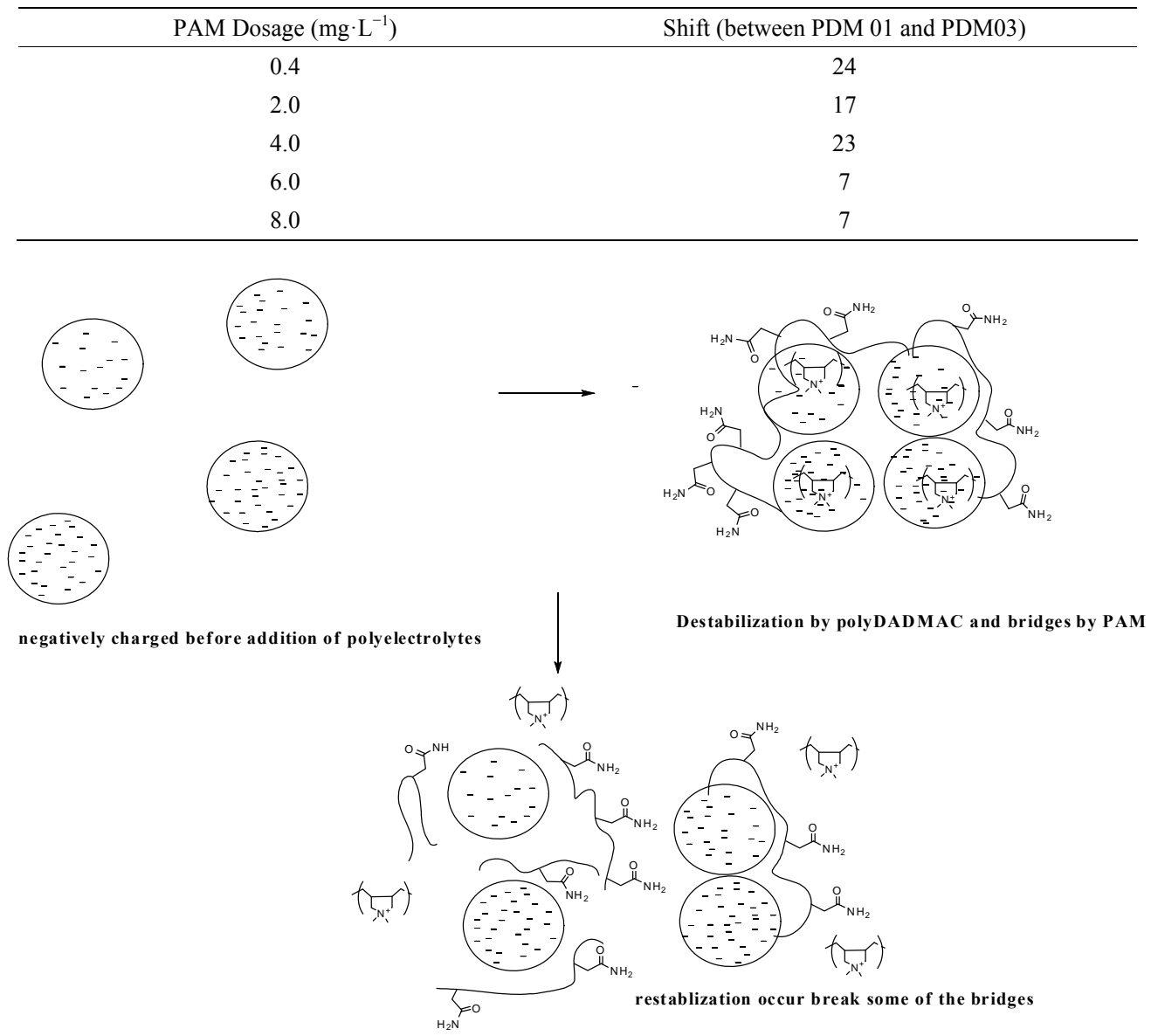

Figure 3. Illustration of saturation and restabilization phenomenon.

of polyDADMAC, with the addition of various PAM dosages, is shown in Figure 4. Turbidity removal and TSS removal results are similar. A log-linear model created by Packman et al. [26] showed a strong positive correlation between TSS and turbidity $\left(\mathrm{R}^{2}=0.96\right)$ with a regression equation of $\ln (\mathrm{TSS})=1.32 \ln (\mathrm{NTU})+\mathrm{C}$ with $\mathrm{C}$ is not significantly different than zero. Approaching the value 1 for " $R$ " " indicates that there is a linear relationship between both results. According to Pavanelli and Bigi [26,27], turbidity is mainly due to particles in suspension. From this figure, the highest molecular weight gives the highest level of TSS removal. Charge neutralization creates condensed particles. In addition, the destabilization process is believed to occur concurrently with a bridging mechanism for higher molecular weights. Increasing molecular weights greatly increases the number of unoccupied absorption sites [28]. Increasing the unoccupied absorption sites increases the tendency for more microflocs to agglomerate. Similar outcomes were observed by Denkov [29], in which the suspended solid removal was higher at higher molecular weights. It was observed that the $1.2 \mathrm{mg} \cdot \mathrm{L}^{-1}$ poly-
DADMAC dosage showed the highest turbidity removal for all PAM dosages, except $4.0 \mathrm{mg} \cdot \mathrm{L}^{-1}$. This indicates that $1.2 \mathrm{mg} \cdot \mathrm{L}^{-1}$ is the optimum dosage for turbidity removal in this system.

The effects of PAM dosage on TSS removal can be split into two stages. The first stage is from $0.4-0.8$ $\mathrm{mg} \cdot \mathrm{L}^{-1}$, while the second stageis from $1.2 \mathrm{mg} \cdot \mathrm{L}^{-1}$ and above. It was observed that the optimal dosage had no significant effect on TSS removal. TSS removal achieved for different PAM dosages with constant molecular weights of polyDADMAC were similar, as shown in Table 3.

Below the polyDADMAC optimal dosage, TSS removal increased with the increasing dosage of PAM. TSS removal started to decrease after a certain dosage, as shown in Table 4. Similar to theturbidity removal results, increasing the PAM dosage increased the probability bridging mechanisms would occur. The increase of bridging increases the size of flocs, hence increasing the amount filtered. The filtered amount is related to TSS removal. Increasing turbidity removal will increase the filtered amount. However, TSS removal decreased with 
dosages higher than $4.0 \mathrm{mg} \cdot \mathrm{L}^{-1}$. This was caused by steric stabilization. At the dosage of $4.0 \mathrm{mg} \cdot \mathrm{L}^{-1}$, the surface particles became saturated and therewere no absorbable sites available for PAM to perform the bridging process. Excessive PAM chains led to steric stabilization and caused the deflocculation of particles in solution, as shown in Figure 5. A part of the PAM segments was strongly absorbed into the particles' surfaces, while other segments were the stabilizing moiety that extends out from the surface into the solution. When two particles approached each other, the extended PAM chains of the stabilizing block came into contact and caused a repulsive force that prevented the particles from aggregating [30].

\subsection{COD Removal}

Figure 6 presents the COD removal from pulp and paper mill wastewater treated with different molecular weights of polyDADMAC, with the addition of PAM in various dosages. Maximum COD removal efficiency was $98.6 \%$, and was achieved by PDM02 at a dosage of $1.2 \mathrm{mg} \cdot \mathrm{L}^{-1}$ and a PAM dosage of $6.0 \mathrm{mg} \cdot \mathrm{L}^{-1}$. It should be noted that COD removal results were in contrast to the turbidity and TSS removal. The highest COD removal was achieved by PDM02, while the best results in regards to turbidity and TSS were achieved by PDM03. However, in all three results, COD removal increased from PDM 01 to PDM 02.

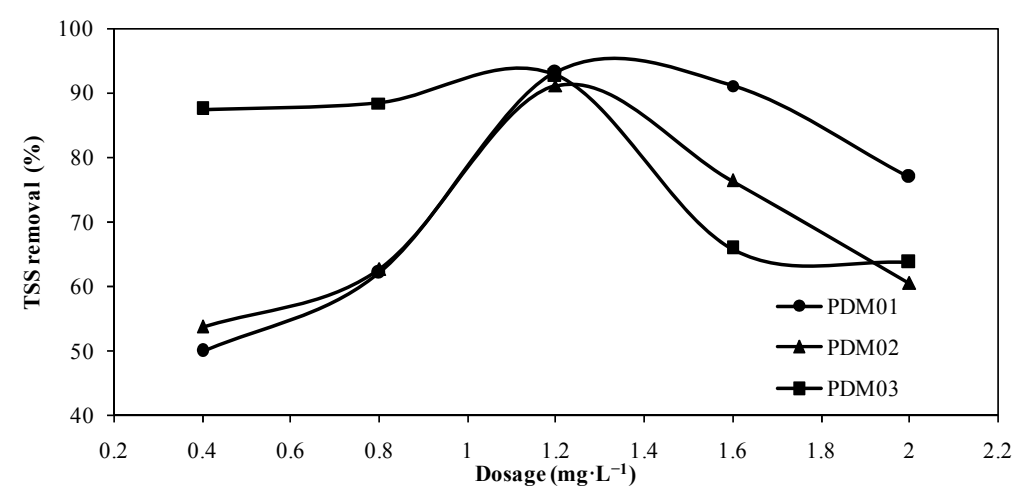

(a)

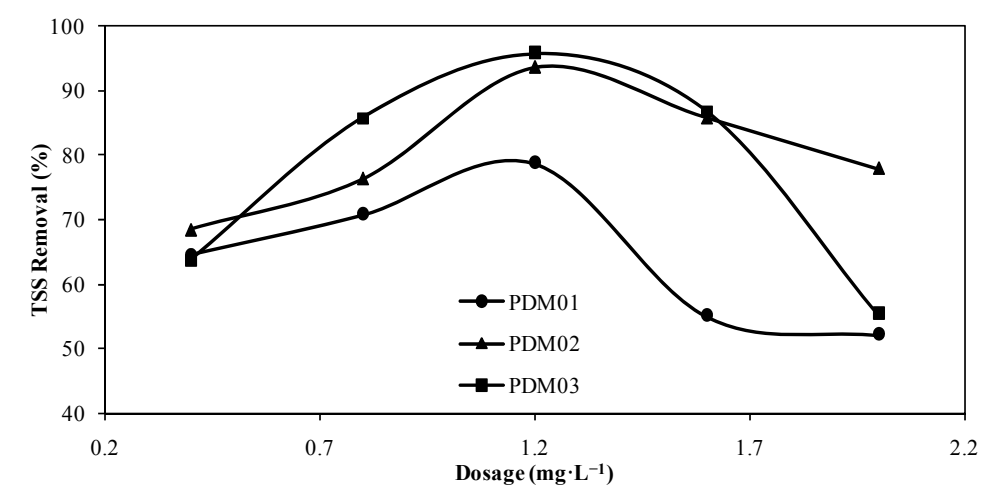

(b)

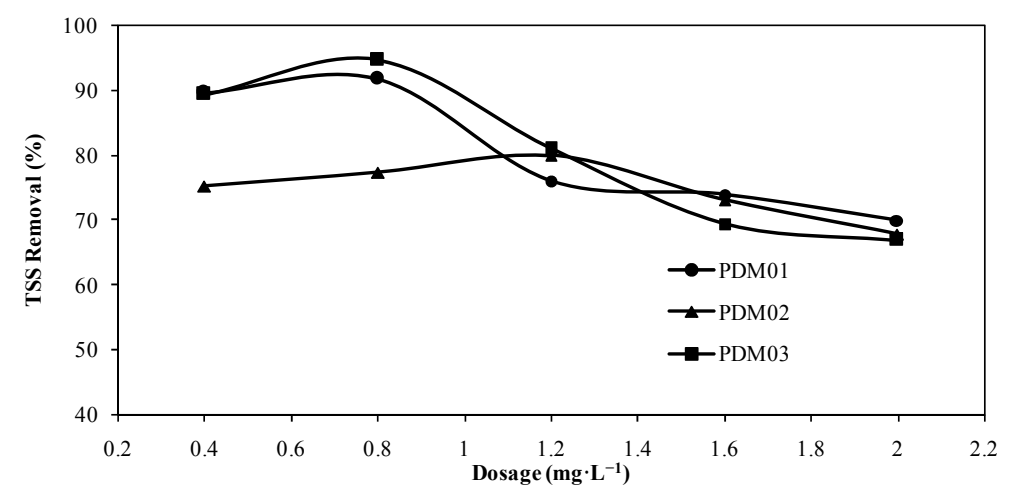

(c) 


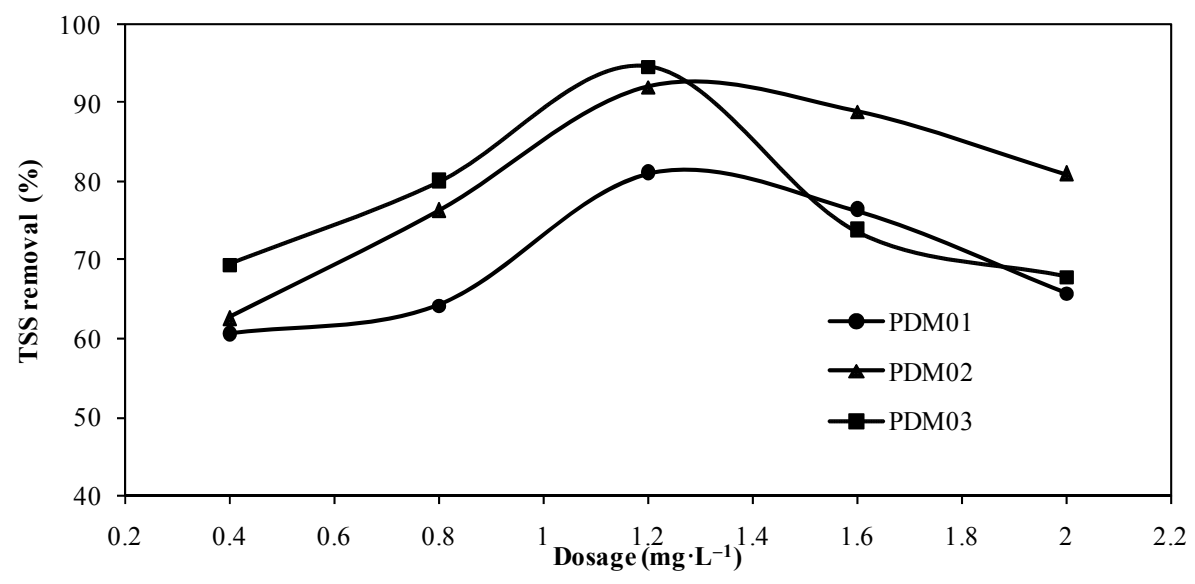

(d)

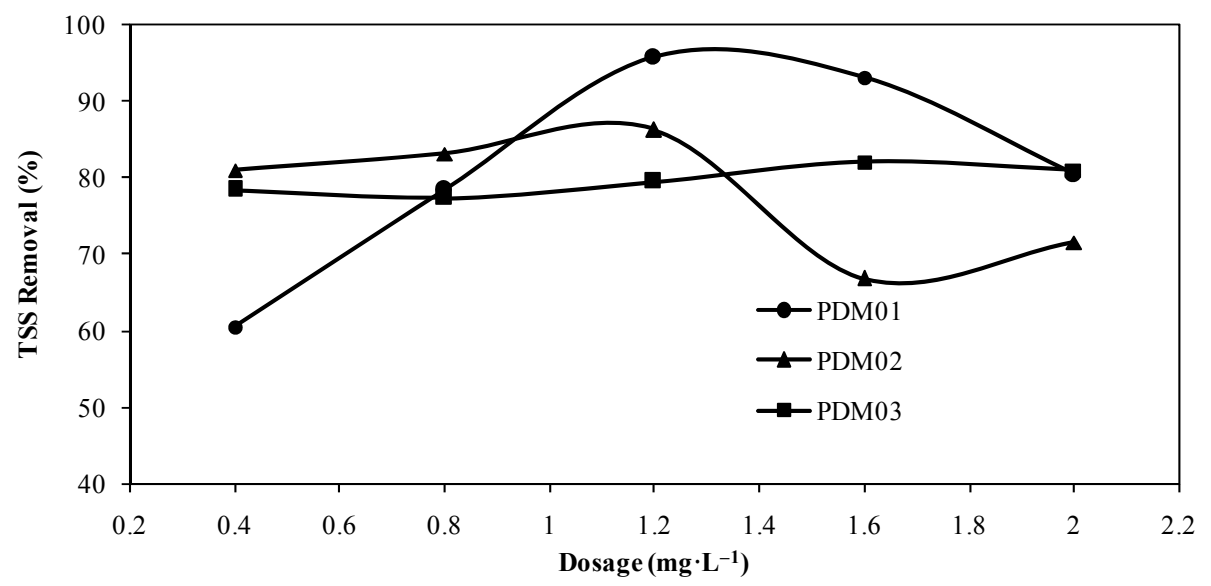

(e)

Figure 4. Effect of polyDADMAC dosage on TSS removal at (a) 0.4 ; (b) 2.0 ; (c) 4.0 ; (d) 6.0 ; and (e) $8.0 \mathrm{PAM} \mathrm{dosages} \mathrm{(mg} \cdot \mathrm{L}^{-1}$ ).

Table 3. TSS removal at optimal dosage of PDM03 with different of PAM dosage.

\begin{tabular}{cc}
\hline PAM Dosage $\left(\mathrm{mg} \cdot \mathrm{L}^{-1}\right)$ & TSS removal $\left(\mathrm{mg} \cdot \mathrm{L}^{-1}\right)$ \\
\hline 0.4 & 92.6 \\
2.0 & 95.8 \\
4.0 & 94.7 \\
6.0 & 94.0 \\
8.0 & 95.8 \\
\hline
\end{tabular}

Table 4. TSS removal at $0.4 \mathrm{mg} \cdot \mathrm{L}^{-1}$ dosage of PDM01 with different of PAM dosage.

\begin{tabular}{cc}
\hline PAM Dosage $\left(\mathrm{mg} \cdot \mathrm{L}^{-1}\right)$ & TSS removal $\left(\mathrm{mg} \cdot \mathrm{L}^{-1}\right)$ \\
\hline 0.4 & 50.0 \\
2.0 & 63.7 \\
4.0 & 75.3 \\
6.0 & 60.5 \\
8.0 & 60.0 \\
\hline
\end{tabular}




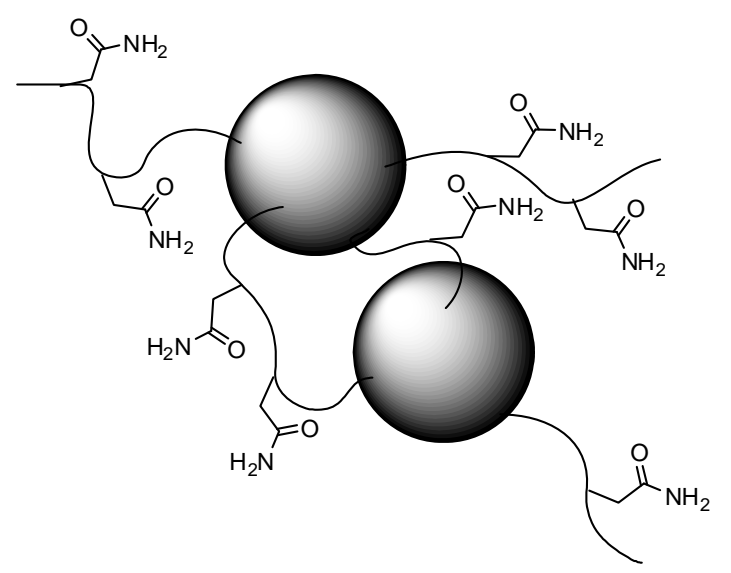

Particles before saturated

(a)

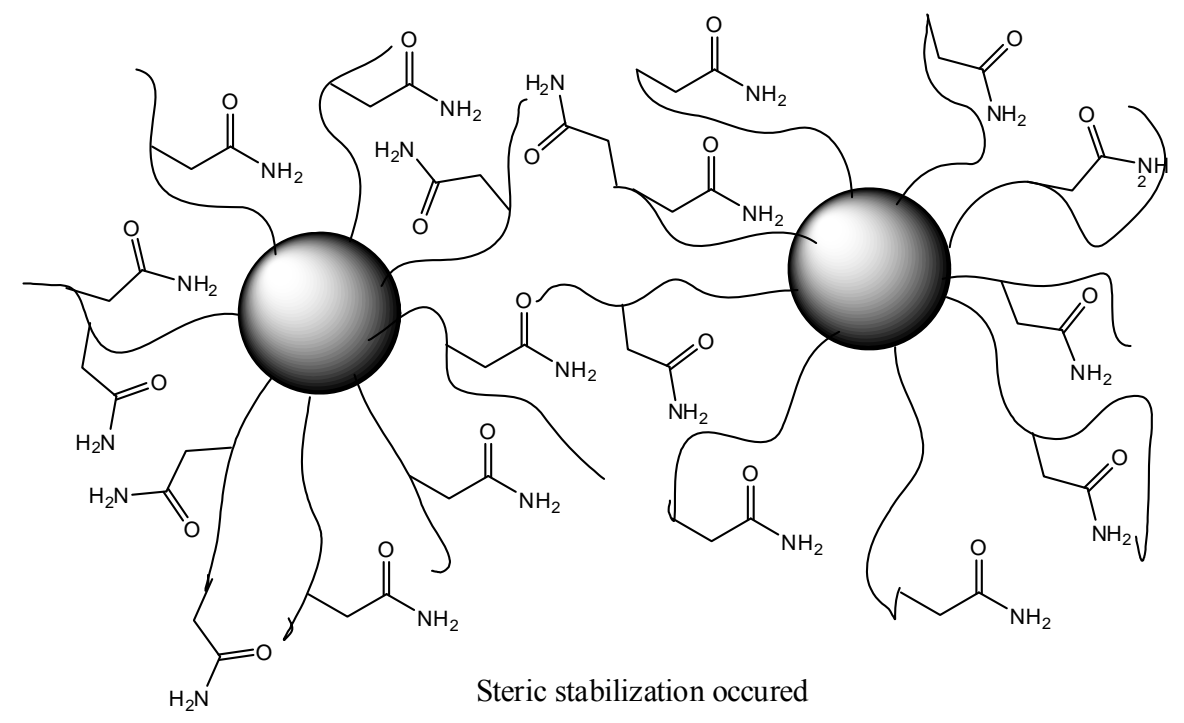

(b)

Figure 5. Illustration particles before saturated and steric stabilization occurred.

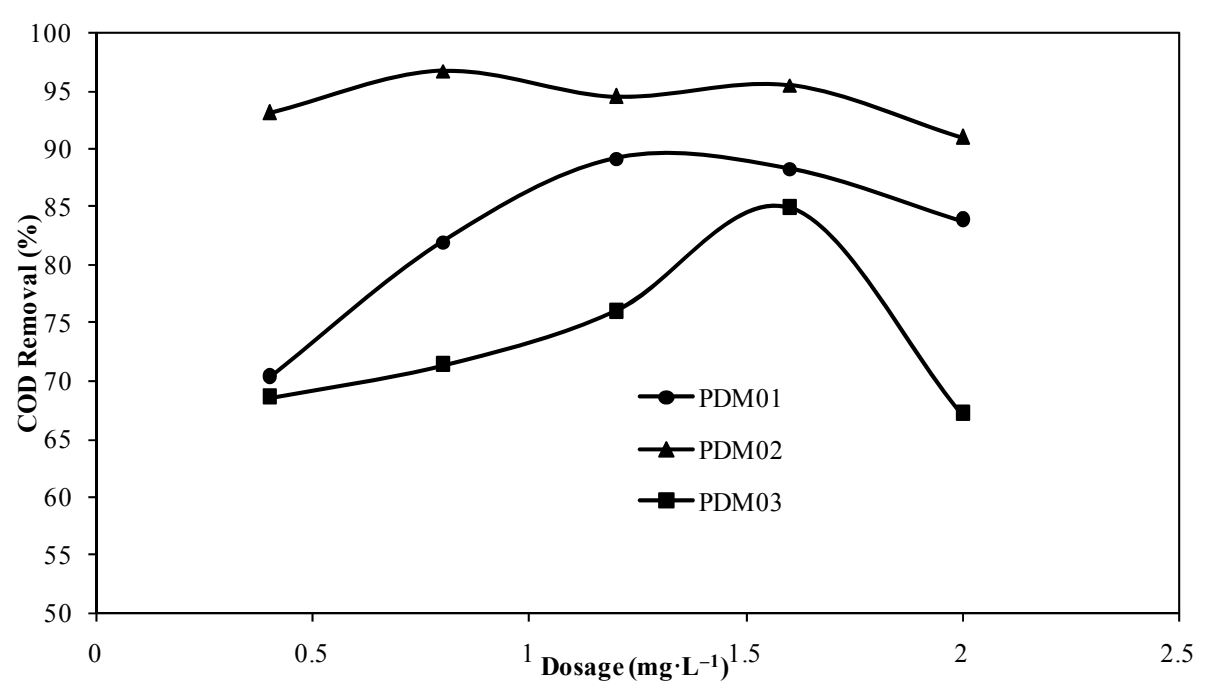

(a) 


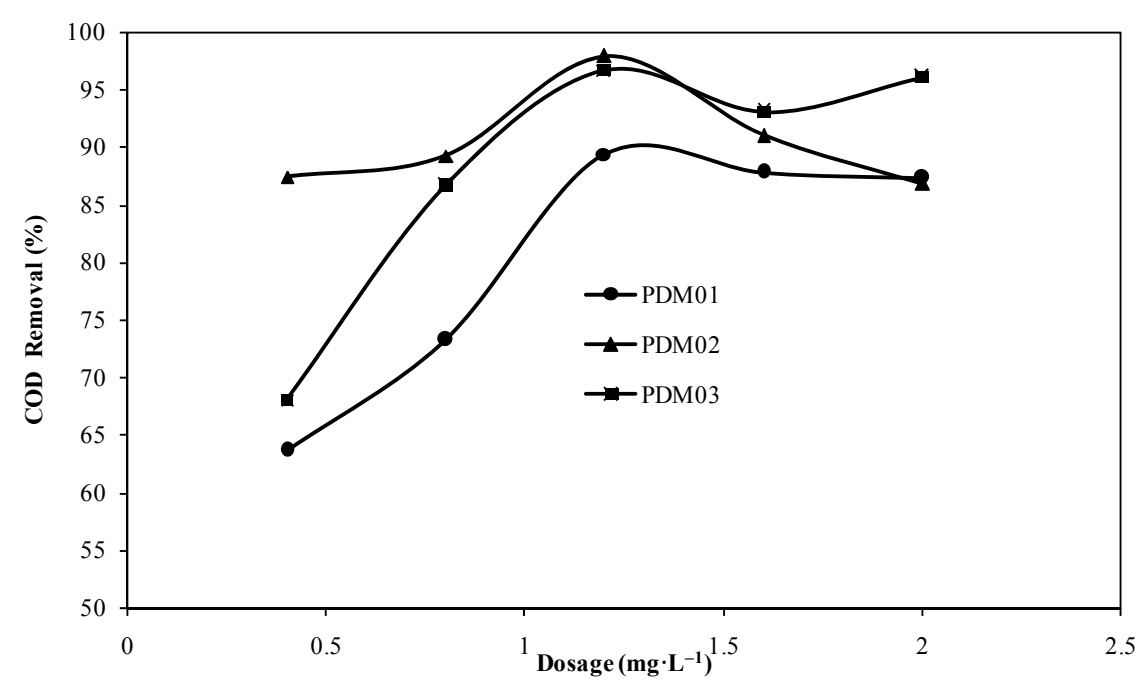

(b)

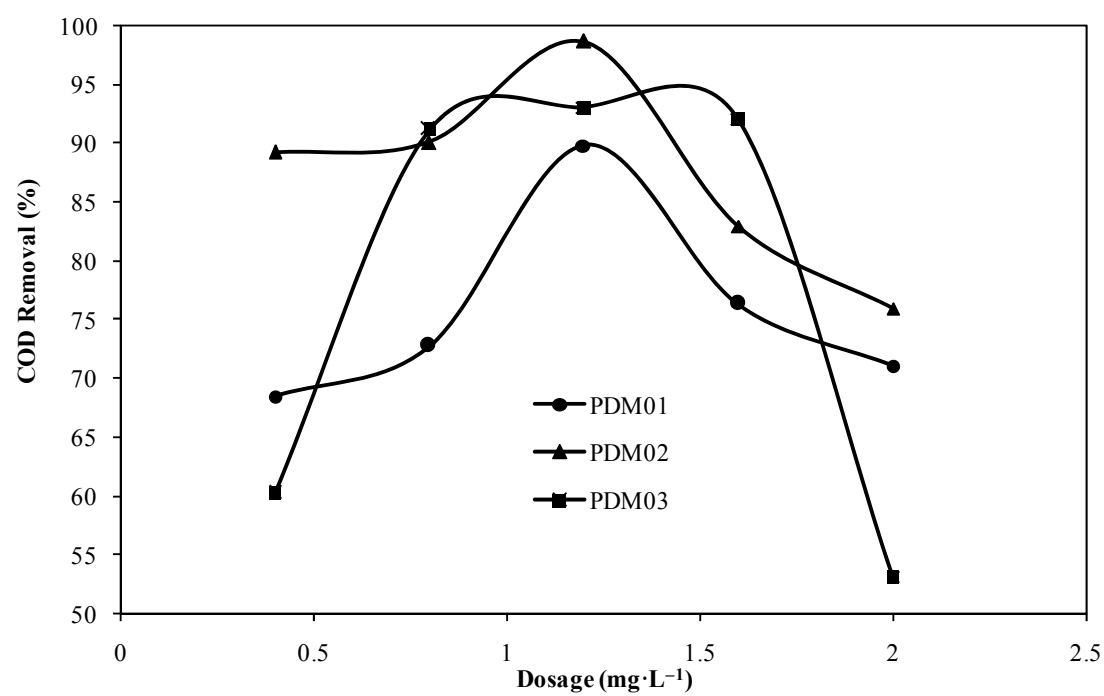

(c)

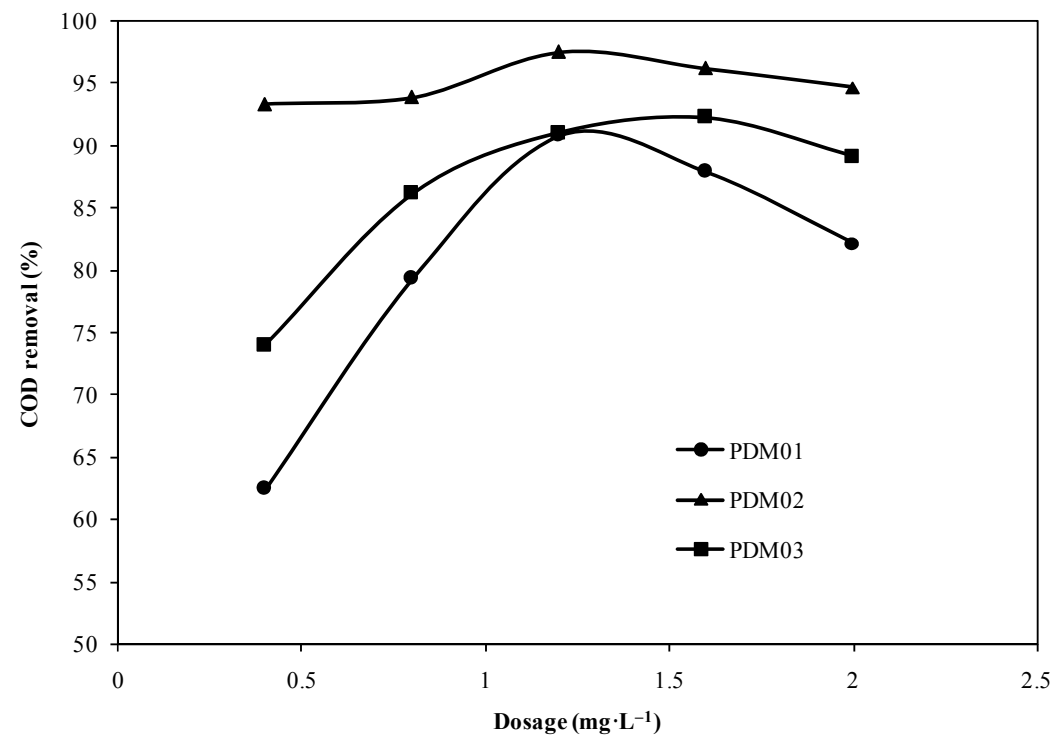

(d) 


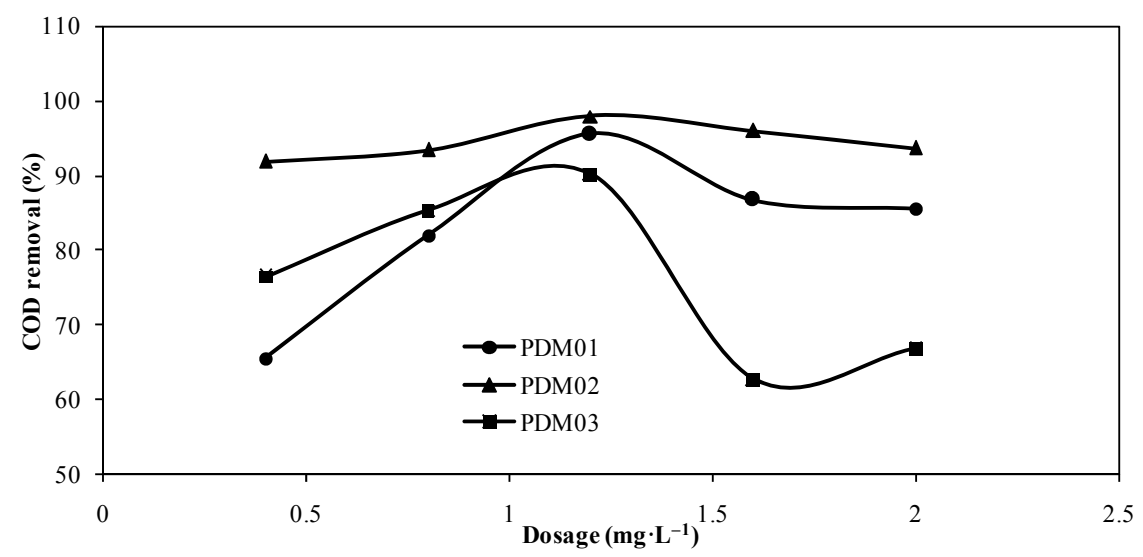

(e)

Figure 6. Effect of PolyDADMACdosages on COD removal at (a) 0.4 ; (b) 2.0 ; (c) 4.0 ; (d) 6.0 ; and (e) 8.0 PAM dosages $\left(\mathbf{m g} \cdot \mathbf{L}^{-1}\right)$.

Generally, COD removal increases with increasing molecular weight, as observed from PDM01 and PDM02. However, the COD removal started to decrease with increasing molecular weights (PDM03 to PDM02). In accordance with the results obtained by Tavares [31], the increase of COD removal from PDM 01 to PDM02 is related to the increase of lignin removal from the wastewater. Decreasing levels of lignin in wastewater reduces oxidation, thus increasing COD removal. The decrease of COD removal from PDM02 to PDM03 is likely caused by polyDADMAC flocculants being discharged along with water, instead of with the suspended solid.

\section{4. $\zeta$ Potential Measurement}

$\zeta$ Potential measurement is an evaluation method for determining the stability and instability of colloidal dispersions. The measurement of $\zeta$ potential is a function of the surface charge of the particles. Particles tend to agglomerate when the value of $\zeta$ potential is closer to zero (isoelectric point) [32]. Figure 7 shows that PDM03 shows a $\zeta$ potential value closer to zero than others. The value closest to zero was achieved at a dosage of 1.2 $\mathrm{mg} \cdot \mathrm{L}^{-1}$ polyDADMAC and a PAM dosage of $8.0 \mathrm{mg} \cdot \mathrm{L}^{-1}$, which was $-0.06 \mathrm{mV}$. This indicates that increasing the molecular weight of PolyDADMAC shifts the $\zeta$ potential value closer to zero. The factors of being more positively charged and higher in molecular weight destabilized the negative charge through van der walls forces. Flocculation started to occur when the attractive van der Waals forces were equal to the repulsive electrostatic force.

However, there was no significant difference in $\zeta$ potential value for different dosages of PAM, as shown in Table 5. PAM has a much less significant effect on $\zeta$ potential compared to polyDADMAC. It is known that $\zeta$ potential is a good indicator of the magnitude of repulsive forces between particles. In this system, the addition of polyDAdMAC destabilized the particles, resulting in big changes in the $\zeta$ potential value, and created microflocs. Many particles became closer to each other, but still few agglomerates formed. Bridgers were created by PAM chains increase the agglomerates formed hence reduced the $\zeta$ potential value a small amount.

PDM 01 and PDM 02 showed positive values after optimal dosing, while PDM 03 showed a negative value with PAM dosages of $0.2 \mathrm{mg} \cdot \mathrm{L}^{-1}$ and $0.2 \mathrm{mg} \cdot \mathrm{L}^{-1}$. PDM 01 and PDM 02 resulted in values of 10.3 and $4.01 \mathrm{mV}$ respectively. Meanwhile, PDM 03 resulted in $-7.75 \mathrm{mV}$. This is possibly contributed to deflocculation. Excessive amounts of polyDADMAC causes charge repulsion, thus creating stability between particles. Meanwhile, excessive amounts of PAM cause saturation, thus breaking the bridges, although microflocs still exist. Zhou and Frank (2006) conclude that the positive value of $\zeta$ potential achieved is contributed to the bridging mechanism, while charge neutralization is more dominant if the $\zeta$ potential achieved is a negative value [33].

\section{Conclusion}

TSSs, the reduction of turbidity, COD removal, and $\zeta$ potential were studied using dual polyelectrolytes as a hybrid flocculant method in treating pulp and paper mill

Table 5. $\zeta$ Potential of wastewater after treatment by different dosage of PAM and constant polyDADMAC dosage at $1.2 \mathrm{mg} \cdot \mathrm{L}^{-1}$.

\begin{tabular}{cc}
\hline PAM Dosage $\left(\mathrm{mg} \cdot \mathrm{L}^{-1}\right)$ & $\zeta$ Potential $(\mathrm{mV})$ \\
\hline 0.4 & -1.12 \\
2.0 & -1.08 \\
4.0 & -1.12 \\
6.0 & -0.06 \\
8.0 & -1.32 \\
\hline
\end{tabular}




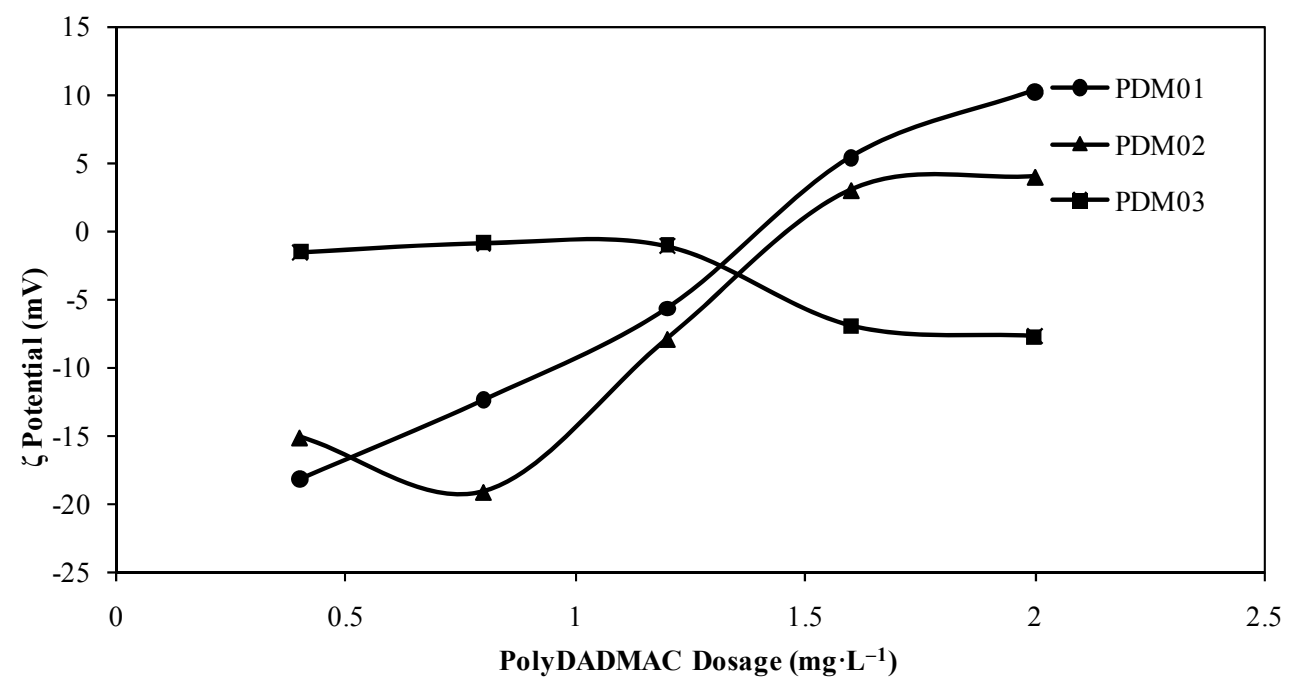

(a)

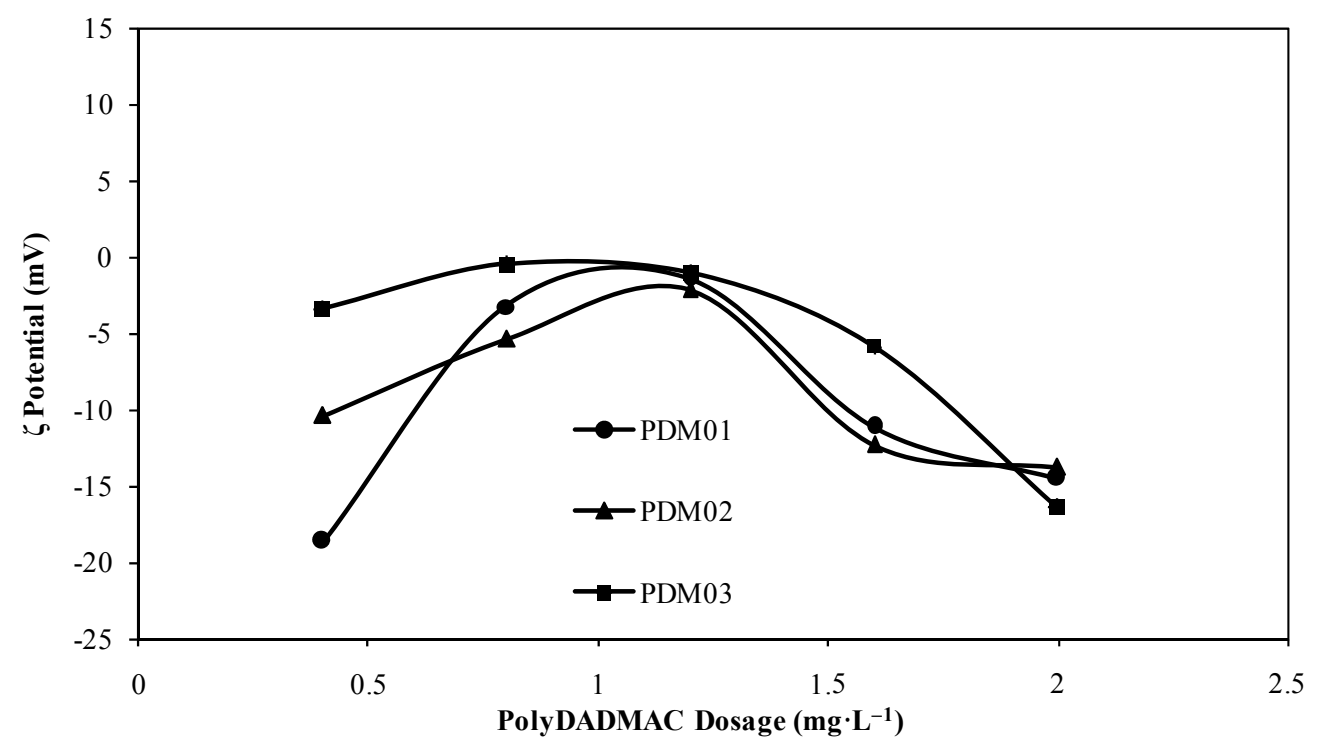

(b)

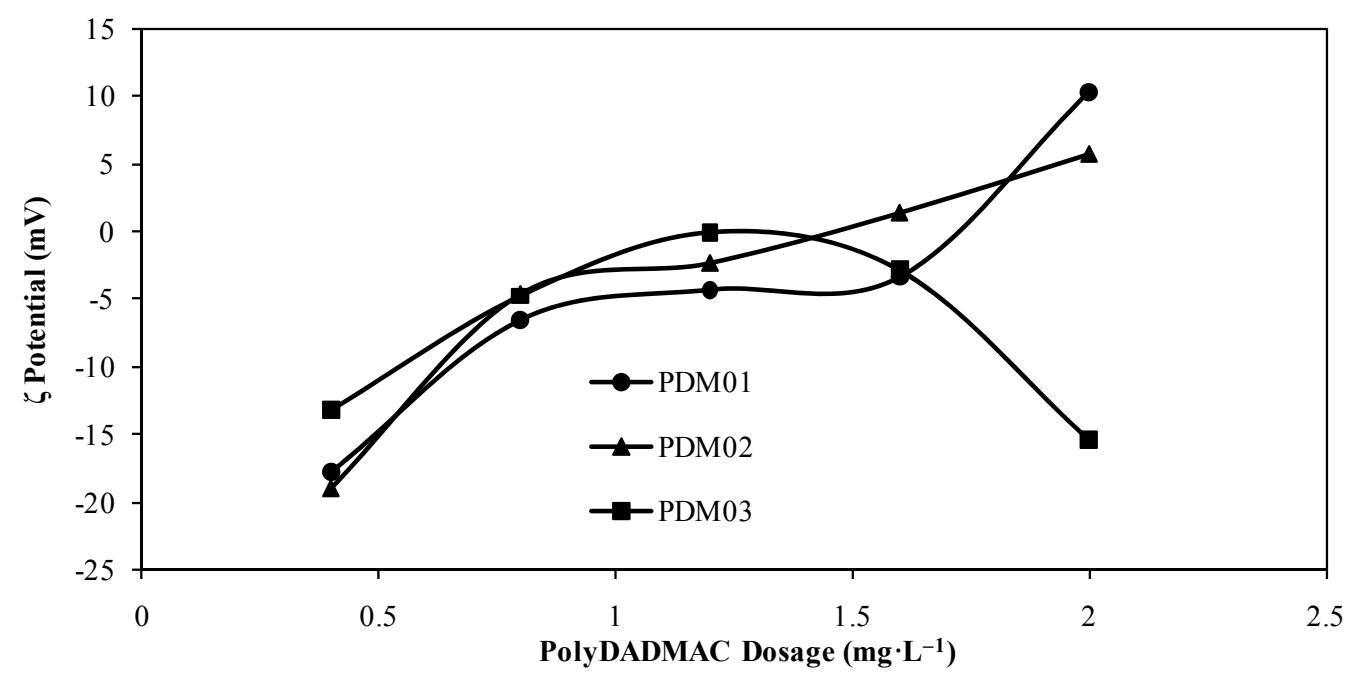

(c) 


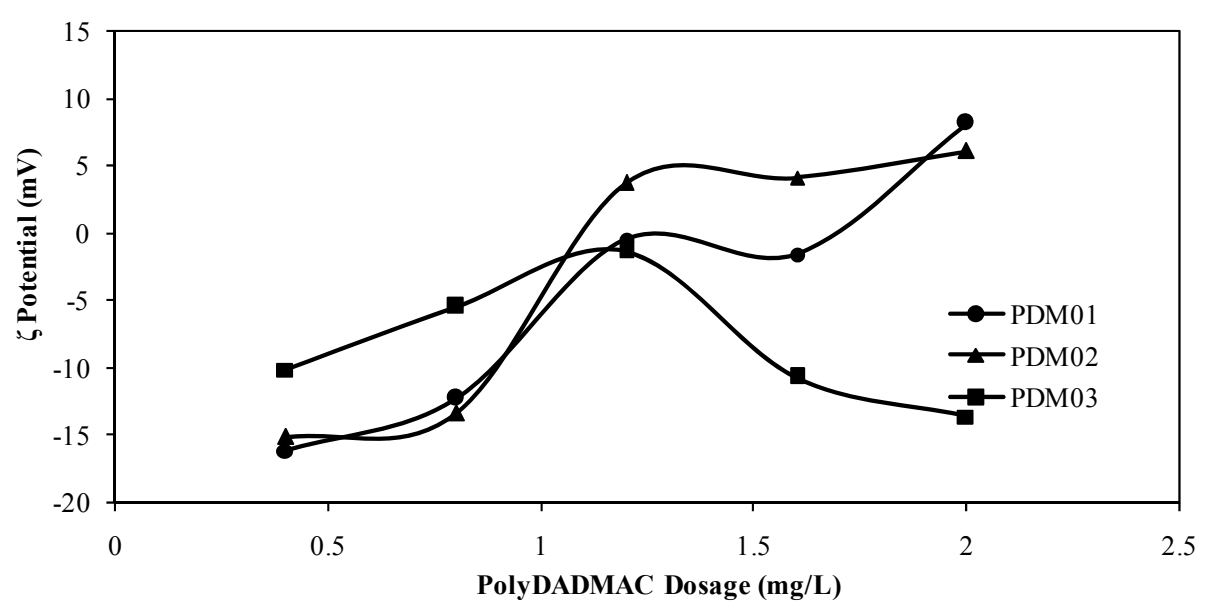

(d)

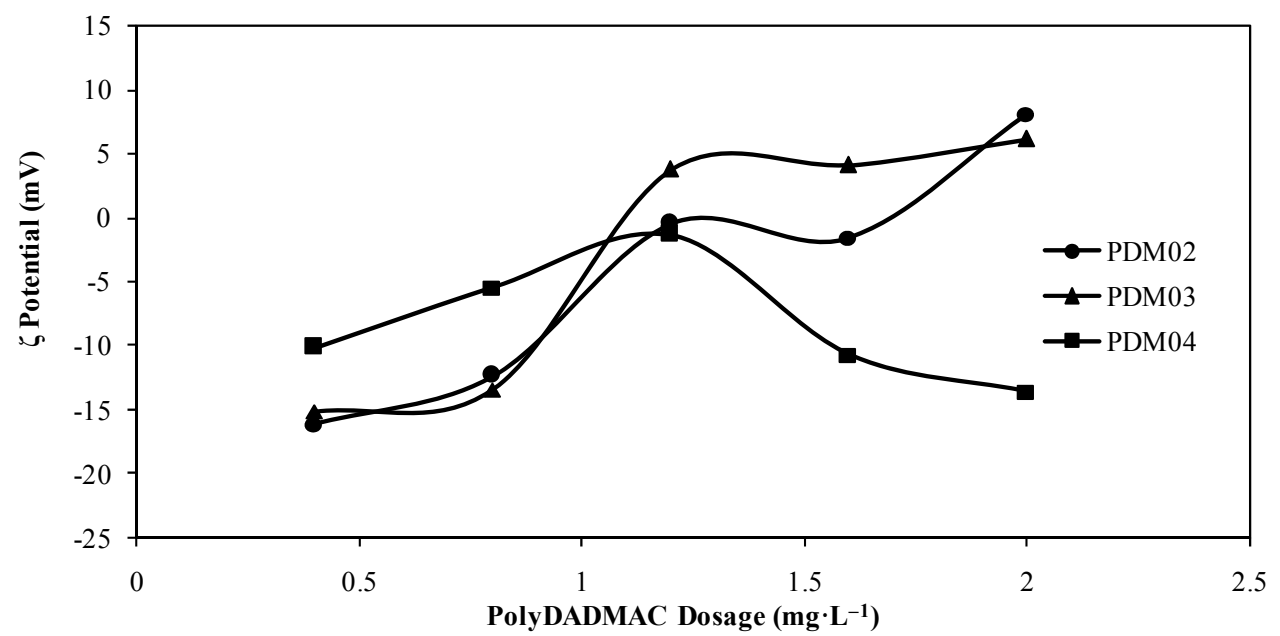

(e)

Figure 7. Effect of PolyDADMAC dosages on $\zeta$ Potential at (a) 0.4 ; (b) 2.0 ; (c) 4.0; (d) 6.0; and (e) 8.0 PAM dosages (mg $\cdot \mathrm{L}^{-1}$ ).

wastewater. In this system, PolyDADMAC functions as a charge destabilizer, while PAM (the loops and tails of the chains link the microflocs) acts as a bridger. Another conclusion is that the highest flocculation occurs at a lower value of the $\zeta$ potential. Decreasing the value of the $\zeta$ potential decreased the repulsive forces between particles, and thus increased the occurrence of flocs.

\section{Acknowledgements}

The authors wish to acknowledge the financial support provided by MOSTI science fund (1001/pbahan/814131) and Global Network Technology (GNT) SdnBhd.

\section{REFERENCES}

[1] H. Tanaka and H. Ichiura, "A Novel and Simple Method for Determining the Cationic Demand of Suspensions Using Chromophoric Labeled Cationic Polymers," Colloids and Surfaces A: Physicochemical and Engineering Aspects, Vol. 159, No. 1, 1999, pp. 103-107.

$$
\text { doi:10.1016/S0927-7757(99)00166-1 }
$$

[2] M. Aguilar, J. Sáez, M. Lloréns, A. Soler and J. F. Ortuño "Microscopic Observation of Particle Reduction in Slaughterhouse Wastewater by Coagulation-Flocculation Using Ferric Sulphate as Coagulant and Different Coagulant Aids," Water Research, Vol. 37, No. 9, 2003, pp. 22332241. doi:10.1016/S0043-1354(02)00525-0

[3] A. L. Ahmad, S. Ismail and S. Bhatia, "Optimization of Coagulation-Flocculation Process for Palm Oil Mill Effluent Using Response Surface Methodology," Environmental Science \& Technology, Vol. 39, No. 8, 2005, pp. 2828-2834. doi:10.1021/es0498080

[4] L.-J. Wang, et al., "A Water-Soluble Cationic Flocculant Synthesized by Dispersion Polymerization in Aqueous Salts Solution," Separation and Purification Technology, Vol. 67, No. 3, 2009, pp. 331-335. doi:10.1016/j.seppur.2009.03.044

[5] Q. Chang, X. K. Hao and L. L. Duan, "Synthesis of Crosslinked Starch-Graft-Polyacrylamide-co-Sodium Xanthate and Its Performances in Wastewater Treatment," Journal of Hazardous Materials, Vol. 159, No. 2-3, 2008, pp. 548-553. doi:10.1016/j.jhazmat.2008.02.053 
[6] X. Yu and P. Somasundaran, "Enhanced Flocculation with Double Flocculants," Colloids and Surfaces A: Physicochemical and Engineering Aspects, Vol. 81, 1993, pp. 1723. doi:10.1016/0927-7757(93)80231-3

[7] A. L. Ahmad, et al., "Improvement of Alum and PACl Coagulation by Polyacrylamides (PAMs) for the Treatment of Pulp and Paper Mill Wastewater," Chemical Engineering Journal, Vol. 137, No. 3, 2008, pp. 510-517. doi:10.1016/j.cej.2007.03.088

[8] M. R. Wu and T. G. M. Van de Ven, "Flocculation and Reflocculation: Interplay between the Adsorption Behavior of the Components of a Dual Flocculant," Colloids and Surfaces A: Physicochemical and Engineering Aspects, Vol. 341, No. 1-3, 2009, pp. 40-45. doi:10.1016/j.colsurfa.2009.03.034

[9] M. Lemanowicz, A. Gierczycki and M. H. Al-Rashed, "Dual-Polymer Flocculation with Unmodified and Ultrasonically Conditioned Flocculant," Chemical Engineering and Processing: Process Intensification, Vol. 50, No. 1, 2010, pp. 128-138.

[10] S. Chitikela and S. K. Dentel, "Dual-Chemical Conditioning and Dewatering of Anaerobically Digested Biosolids: Laboratory Evaluations," Water Environment Research, Vol. 70, No. 5, 1998, pp. 1062-1069. doi:10.2175/106143098X123408

[11] D. Tao, J. G. Groppo and B. K. Parekh, "Enhanced Ultrafine Coal Dewatering Using Flocculation Filtration Processes," Minerals Engineering, Vol. 13, No. 2, 2000, pp. 163-171. doi:10.1016/S0892-6875(99)00162-4

[12] E. Sabah, H. Yuzer and M. S. Celik, "Characterization and Dewatering of Fine Coal Tailings by Dual-Flocculant Systems," International Journal of Mineral Processing, Vol. 74, No. 1-4, 2004, pp. 303-315. doi:10.1016/j.minpro.2004.03.001

[13] S. Mathur, P. Singh and B. Moudgil, "Advances in Selective Flocculation Technology for Solid-Solid Separations," International Journal of Mineral Processing, Vol. 58, No. 1-4, 2000, pp. 201-222. doi:10.1016/S0301-7516(99)00072-1

[14] A. Swerin, L. Ödberg and L. Wågberg, “An Extended Model for the Estimation of Flocculation Efficiency Factors in Multicomponent Flocculant Systems," Colloids and Surfaces A: Physicochemical and Engineering Aspects, Vol. 113, No. 1-2, 1996, pp. 25-38. doi:10.1016/0927-7757(95)03506-0

[15] B.-U. Cho, et al., "A Bridging Model for the Effects of a Dual Component Flocculation System on the Strength of Fiber Contacts in Flocs of Pulp Fibers: Implications for Control of Paper Uniformity," Colloids and Surfaces A: Physicochemical and Engineering Aspects, Vol. 287, No. 1-3, 2006, pp. 117-125. doi:10.1016/j.colsurfa.2006.03.029

[16] K. Britt, "Retention of Additives during Sheet Formation," Tappi, Vol. 56, No. 3, 1973, pp. 83-86.

[17] A. Swerin, G. Glad-Nordmark and L. Odberg, "Adsorption and Flocculation in Suspensions by Two Cationic Polymers: Simultaneous and Sequential Addition," Journal of Pulp and Paper Science, Vol. 23, No. 8, 1997, pp. J389-J393.
[18] A. C. Rodrigues, et al., "Treatment of Paper Pulp and Paper Mill Wastewater by Coagulation-Flocculation Followed by Heterogeneous Photocatalysis," Journal of Photochemistry and Photobiology A: Chemistry, Vol. 194, No. 1, 2008, pp. 1-10. doi:10.1016/j.jphotochem.2007.07.007

[19] S. Wong, et al., "Treatment of Pulp and Paper Mill Wastewater by Polyacrylamide (PAM) in Polymer Induced Flocculation," Journal of Hazardous Materials, Vol. 135, No. 1-3, 2006, pp. 378-388. doi:10.1016/j.jhazmat.2005.11.076

[20] M. A. A. Razali, et al., "Treatment of Pulp and Paper Mill Wastewater with Various Molecular Weight of PolyDADMAC Induced Flocculation," Chemical Engineering Journal, Vol. 166, No. 2, 2011, pp. 529-535. doi:10.1016/j.cej.2010.11.011

[21] A. McFarlane, et al., "The Influence of Flocculant Adsorption Kinetics on the Dewaterability of Kaolinite and Smectite Clay Mineral Dispersions," Colloids and Surfaces A: Physicochemical and Engineering Aspects, Vol. 317, No. 1-3, 2008, pp. 39-48. doi:10.1016/j.colsurfa.2007.09.045

[22] R. Subramanian, S. Zhu and R. Pelton, "Synthesis and Flocculation Performance of Graft and Random Copolymer Microgels of Acrylamide and Diallyldimethylammonium Chloride," Colloid \& Polymer Science, Vol. 277, No. 10, 1999, pp. 939-946. doi:10.1007/s003960050473

[23] R. Shatat, et al., "The Effect of Molecular Weight and Charge Density on Floc Size Distribution of Palm Oil Effluent Flocculated with Cationic Polyelectrolytes," Journal of Basic and Applied Sciences, Vol. 4, No. 2, 2008, pp. 95-103.

[24] X. H. Zhang, et al., "Flocculation of Reed Pulp Suspensions by Quaternary Chitosan Nanoparticle $\mathrm{SiO}_{2}$ Retention Aid Systems," Journal of Applied Polymer Science, Vol. 117, No. 2, 2010, pp. 742-749. doi:10.1002/app.30230

[25] B. J. Lee, "Experimental and Modeling Studies for Optimizing Flocculant-Aided Sediment Retention Ponds," Clemson University, Clemson, 2008.

[26] J. Packman, K. Comings and D. Booth, "Using Turbidity to Determine Total Suspended Solids in Urbanizing Streams in the Puget Lowlands," Confronting Uncertainty: Managing Change in Water Resources and the Environment, Canadian Water Resources Association Annual Meeting, Vancouver, 27-29 October 1999, pp. 158-165.

[27] D. Pavanelli and A. Bigi, "Indirect Methods to Estimate Suspended Sediment Concentration: Reliability and Relationship of Turbidity and Settleable Solids," Biosystems Engineering, Vol. 90, No. 1, 2005, pp. 75-83.

[28] C.-Y. Yin, "Emerging Usage of Plant-Based Coagulants for Water and Wastewater Treatment," Process Biochemistry, Vol. 45, No. 9, 2010, pp. 1437-1444. doi:10.1016/j.procbio.2010.05.030

[29] N. Denkov, et al., "Mechanism of Formation of Two-Dimensional Crystals from Latex Particles on Substrates," Langmuir, Vol. 8, No. 12, 1992, pp. 3183-3190. doi:10.1021/la00048a054

[30] C. Tripp and M. Hair, "Controlled Flocculation-Deflocculation Behavior of Adsorbed Block Copolymers in 
Colloidal Dispersions by Modifying Segment/Surface Interactions: The Use of Small Displacer Molecules to Selectively Cleave Interparticle Bonds," Langmuir, Vol. 10, No. 11, 1994, pp. 4031-4038. doi:10.1021/la00023a022

[31] C. Tavares, et al., "Ultrafiltration/Complexation Process for Metal Removal from Pulp and Paper Industry Wastewater," Desalination, Vol. 144, No. 1-3, 2002, pp. 261265. doi:10.1016/S0011-9164(02)00325-9
[32] L. C. Li and Y. Tian, "Encyclopedia of Pharmaceutical Technology," In: Zeta Potential, 2nd Edition, Marcel Dekker, Inc., New York, 2002, p. 3020.

[33] Y. Zhou and G. V. Franks, "Flocculation Mechanism Induced by Cationic Polymers Investigated by Light Scattering," Langmuir, Vol. 22, No. 16, 2006, pp. 6775-6786. doi:10.1021/la060281+ 